\title{
Efeito antimicrobiano do extrato de cranberry sobre micro-organismos causadores de infecção urinária
}

\section{Antimicrobial effect of cranberry extract on microorganisms that cause urinary infection}

1 Carla Franco Porto Belmont Souza carla.belmont@outlook.com

2 Luiz Eduardo Souza da Silva Irineu

2 Renan Silva de Souza

3 Renato da Silva Teixeira

4 Ivina Sanches Pereira

${ }^{5}$ Carlos Alberto Sanches Pereira

\footnotetext{
Discente do curso de Ciências Biológicas, UniFOA.

Graduado em Ciências Biológicas, UniFOA.

Associação Educacional Dom Bosco, AEDB.

Universidade Federal Fluminense, UFF.

Docente do Centro Universitário de Volta Redonda, UniFOA.
}

\section{RESUMO}

A resistência microbiana tem se mostrado um problema de proporções mundiais, causando estado de morbidade e mortalidade em diversos pacientes. Em vista disso, tem crescido a busca por métodos alternativos naturais de profilaxia. A investigação clínica sugere que o Extrato de Cranberry está entre as melhores propostas de prevenção natural. O Cranberry (Vaccinium macrocarpon) é um fruto que tem crescido comercialmente pelo sabor e propriedades benéficas à saúde. Dentre as formas comercializadas estão: o suco, o chá e as cápsulas contendo o extrato seco. A ação desta planta está relacionada ao tratamento de doenças do trato urinário, por possuir substâncias que inibem a adesão bacteriana ao epitélio do trato urinário, dificultando sua proliferação e reprodução. Dentre todas as infecções relacionadas à assistência a saúde, a Infecção do Trato Urinário é a mais frequentemente associada a procedimentos invasivos. Se não for tratada, pode resultar em complicações como pielonefrite aguda, bacteremia e pionefrose. Portanto, cranberry pode ser uma nova.

\section{Palavras-chave}

extrato de cranberry, infecção urinária, Vaccinium macrocarpon.

\begin{abstract}
The microbial resistance has been a problem of world proportions, causing morbidity state and mortality in several patients. Regarding this, the search for natural alternative methods of prophylaxis has been growing. The clinical investigation suggests that the Cranberry Extract is between the best natural prevention proposals. The Cranberry (Vaccinium macrocarpon) is a fruit that has been growing commercially because of its flavor and the beneficial health properties. Among the commercialized types are: the juice, the tea and the pills containing the dry extract. This plant's action is related to the treatment of urinary tract diseases, because it contains substances that inhibit the bacterial adhesion to the urinary tract's epithelium, making it difficult for them to proliferate and reproduce. Among all the infections related to health assistance, the Urinary Tract Infection is the most frequently associated to invasive procedures. If it is not treated, could result in complications like acute pyelonephritis, bacteremia and pyonephrosis. Therefore, the cranberry could be an alternative.
\end{abstract}

\section{Keywords}

cranberry extract, urinary tract infection, Vaccinium macrocarpon.

\section{Como você deve citar?}

SOUZA, Carla Franco Porto Belmont. Efeito antimicrobiano do extrato de cranberry sobre micro-organismos causadores de infecção urinária. Cadernos UniFOA, Volta Redonda, n. 31, p. 113-122, ago. 2016. 


\section{INTRODUÇÃO}

Desde a descoberta da penicilina, em 1928, por Alexander Fleming, os antimicrobianos vêm sendo usados em larga escala, como forma de combate aos micro-organismos nocivos a fim de tornar melhor a condição da saúde humana. Porém, o uso demasiado destas drogas exerce pressão seletiva sobre estes micro-organismos, que por sua vez, desenvolvem mecanismos de defesa contra elas, tornando-se resistentes.

A resistência bacteriana tem se mostrado um problema de proporções mundiais, causando estado de morbidade e mortalidade em pacientes pós-cirúrgicos, em uso de quimioterapia, com imunodeficiências adquiridas e congênitas e também a indivíduos não hospitalizados, que estão expostos a diversos micro-organismos resistentes (LIVERMORE, 2003; GARZA-RAMOS; SILVA-SÁNCHEZ; MARTÍNEZROMERO, 2009). Em vista disso, tem crescido a busca por métodos alternativos naturais de profilaxia.

Entre os métodos alternativos, o uso medicinal das plantas tem se mostrado de grande eficácia na medicina desde a antiguidade. A organização Mundial de Saúde (OMS) tem incentivado fortemente desde 1977 o estudo de plantas medicinais, com o intuito de avaliar os benefícios do uso de fitoterápicos (LOGUERCIO et al., 2005). A investigação clínica sugere que o Extrato de Cranberry está entre as melhores propostas de prevenção natural (HEAD, 2008).

O cranberry (Vaccinum macrocarpon) é um fruto da família Ericaceae, originário da América do Norte e cresce em pântanos ácidos cheios de musgos de trufas em florestas úmidas (SMALL, 2013). Antigamente, era utilizado como conservante de carne de alce; tinta natural na coloração das vestimentas, cobertores e tapetes; e, também se usava em ferimentos causados por flechas envenenadas, devido à crença em seu efeito antisséptico. Atualmente, o Cranberry tem crescido comercialmente pelo seu sabor e também por suas propriedades benéficas à saúde. Dentre as formas comercialmente ativas estão presentes: o suco, o chá e as cápsulas contendo o extrato seco. Existem outros membros pertencentes do gênero Vaccinium que compartilham alguns de seus componentes básicos, como 0 Cranberry Europeia (Vaccinium Oxycoccus); Lingonberry (Vaccinium vitisidaea); mirtilo (Vaccinium myrtillus) (HISANO et al., 2012). Dentre as substâncias benéficas presentes em sua composição, pode-se citar proantocianidinas; ácido cumarínico; ácidos fenólicos, como o ácido benzoico e o ácido caféico; flavonóides, como quercetina e miricetina (CHEN; ZUO; DENG, 2001).

O gênero Vaccinum tem sido usado como remédio popular para prevenir infecções do trato urinário durante anos. Em 1984 foi descoberto que o cranberry interfere na adesão das bactérias às células uroepiteliais, evitando assim as infecções. Em 1989 foram identificadas as proantocianidinas (PACs), um dos principais compostos presentes no cranberry capaz de inibir a aderência da Escherichia coli com P-fímbrias à mucosa urogenital (GUAY, 2009; WANG et al., 2012). As substâncias presentes na planta com potencial de inibição interferem na proliferação e reprodução dos patógenos (HISANO et al., 2012).

Estudos mostraram que o extrato de cranberry europeu apresenta atividade antimicrobiana ao ser capaz de inibir o crescimento de micro-organismos patogênicos, tanto gram-positivos quanto gram-negativos (ČESONIENE்; JASUTIENE்; ŠARKINAS, 2009). Dentre os micro-organismos mais frequentes na infecção ao trato urinário, um dos principais é a bactéria Escherichia coli uropatogênica (UPEC). Essa infecção ocorre principalmente em mulheres, que possuem a uretra mais curta, facilitando a entrada de micro-organismos (HOWELL et al., 2010; DEBNATH et al., 2013). Em adição, o cranberry pode reduzir significativamente a aderência de outros patógenos comuns do trato urinário, incluindo Proteus mirabilis, Staphylococcus aureus, Klebsiella pneumoniae, Enterobacter, Pseudomonas aeruginosa, Enterococcus spp. e Candida spp. (MAGARINÕS et al., 2008; EROL; ÇOBAN; TEKIN, 2014). 
De acordo com os estudos de Armbruster; Mobley (2012), certos mecanismos estão presentes na entrada e estabelecimento de alguns micro-organismos no trato urinário, como fímbrias, adesinas, urease e translocação bacteriana. Além da possibilidade de modulação da expressão dos fatores de adesão e de motilidade, que são importantes para a ascensão e produção de fatores de virulência especializados para a sobrevivência no interior do trato urinário. A estrutura proteica principal relacionada com a adesão é a proteína adesina fímbrial, que é inibida por frutose (presente em uvas, laranjas, e cranberries) (HISANO et al., 2012).

Vários estudos mostraram que as características da superfície e a expressão de genes das bactérias podem ser alteradas pelo cranberry, influenciando no processo inicial da adesão bacteriana (AHUJA; KAACK; ROBERTS, 1998; LIU et al., 2006, 2008; JOHNSON et al. 2008). A atuação das proantocianidinas do cranberry também foi demonstrada em Staphylococcus aureus e Staphylococcus aureus resistente à meticilina (MRSA), de modo que a mesma tem capacidade de causar danos à parede celular. Essa capacidade foi observada ao se constatar vários genes supra-regulados envolvidos na biossíntese da parede celular, semelhante à supra-regulação causada por antibióticos conhecidos por interromper a biossíntese de peptideoglicanos (SU; HOWELL; D'SOUZA, 2012; DIARRA et al., 2013). 0 cranberry também pode atuar, em adição a outros taninos, no bloqueio da motilidade e translocação bacteriana em Pseudomonas aeruginosa (O'MAY; TUFENKJI, 2011).

Dentre todas as infecções relacionadas à assistência a saúde (IRAs), a infecção do trato urinário (ITU) tem recebido grande incentivo para seu controle por causa de sua alta ocorrência e medidas preventivas existentes. Contudo, se não for prevenida ou resolvida, resulta em complicações, que podem incluir o desenvolvimento de pielonefrite aguda, bacteremia e pionefrose. Sendo que a sua incidência está constantemente relacionada com os procedimentos invasivos (SOUZA et al., 2007; ARMBRUSTER et al., 2014; EROL; ÇOBAN; TEKIN, 2014).

Um dos procedimentos invasivos associados ao aumento da frequência de ITU é o cateterismo urinário, que está relacionado ao desenvolvimento de bacteremia e bacteriúria. 0 estabelecimento de bacteriúria se deve ao biofilme formado ao longo da superfície do cateter. Após a inserção do cateter, a formação do biofilme ao longo da superfície se inicia imediatamente, quando os organismos aderem a um filme de condicionamento de proteínas do hospedeiro (NICOLLE, 2014).

Estudos sobre a eficácia na aplicabilidade do Cranberry mostraram que pode ser utilizado em caso de cistite recorrente; e, poderá ser associado com antibiótico no tratamento de infecção do trato urinário aguda (DESSì; ATZEl; FANOS, 2011).

Sendo assim, cranberry pode ser uma nova alternativa para o combate das infecções uroepiteliais, já que este é um produto natural de fácil acesso, e com formas de comercialização diversificadas, ao contrário dos antimicrobianos convencionais, que por sua vez são caros e podem acabar causando resistência nos micro-organismos.

Este trabalho teve como objetivo avaliar in vitro a atividade antimicrobiana do extrato de Cranberry sobre 8 bactérias isoladas de infecções urinárias de pacientes internados em unidades de Saúde.

\section{MATERIAL E MÉTODOS}

O experimento foi realizado nos Laboratórios de Biotecnologia e Microbiologia do Centro Universitário de Volta Redonda - UniFOA, Volta Redonda, RJ. 


\subsection{Aquisição dos micro-organismos}

As bactérias foram adquiridas do Instituto Nacional de Controle de Qualidade em Saúde (INCQS) (Coleção de Micro-organismos de Referência do Instituto Oswaldo Cruz IOC/FIOCRUZ), sendo todas isoladas de pacientes com infecções urinárias.

As cepas utilizadas foram: Escherichia coli, Pseudomonas aeruginosa, Klebsiella pneumoniae, Proteus mirabilis, Serratia marscecens, Staphylococcus aureus, Enterococcus faecalis e Enterococcus faecium.

\subsection{Obtenção do extrato de Cranberry}

O extrato de cranberry foi adquirido em loja especializada de produtos naturais do município de Volta Redonda e foi armazenado de acordo com as instruções do fornecedor.

O extrato foi hidratado com água destilada a fim de se obter a concentração de $64 \mathrm{mg} / \mathrm{mL}$ de proantocianidinas (PACs). A concentração mencionada foi utilizada por se encontrar entre 36 e $108 \mathrm{mg} /$ $\mathrm{mL}$ de proantocianidinas (PACs), que corresponde às doses estimadas atualmente para a prevenção de infecções do trato urinário (LAVIGNE et al., 2008).

\subsection{Armazenamento das Cepas}

Os micro-organismos foram devidamente armazenados conforme protocolo obtido do fornecedor das cepas.

Todas as amostras foram devidamente armazenadas em criotubos contendo caldo Brain Heart Infusion (BHI) com glicerol (20\%) e mantidas a $-20^{\circ} \mathrm{C}$ no freezer.

\subsection{Avaliação de Atividade Antimicrobiana do Cranberry}

O estudo foi feito em tubos de ensaio contendo caldo Mueller Hinton (MH), Extrato de Cranberry e as bactérias patogênicas. 0 ensaio se deu com a utilização de um controle de crescimento das bactérias em meio $\mathrm{MH}$ e o experimento para avaliação do crescimento bacteriano na presença do extrato de Cranberry, conforme Figura 1.

Para analisar o efeito do extrato, as bactérias foram ativadas anteriormente em caldo $\mathrm{MH}$, sendo então incubadas à $37^{\circ} \mathrm{C}$ por 24 horas. Este processo foi realizado durante três dias consecutivos, para que as células bacterianas estivessem com seu metabolismo ativo. Após esta etapa, padronizou-se 0 inóculo com a escala de McFarland número 1 (3,0 x 10 $\mathrm{UFC} / \mathrm{mL})$.

0 ensaio foi realizado em triplicata, expondo cada bactéria ao extrato de Cranberry. Para isso, utilizou-se $2 \mathrm{~mL}$ da suspensão de cada bactéria em tubo de ensaio estéril e, em seguida, adicionou-se $2 \mathrm{~mL}$ de extrato de cranberry.

A turbidez foi medida com o auxílio de um espectrofotômetro FEMTO 700 Plus no comprimento de onda de $600 \mathrm{~nm}$. Para realizar a espectrofotometria, foi retirado $1 \mathrm{~mL}$ de cada tubo antes e após 24 horas de incubação à $37^{\circ} \mathrm{C}$. 0 procedimento forneceu a Densidade Ótica (DO), do qual possibilitou a identificação do potencial inibitório do Cranberry sobre as diversas bactérias utilizadas. 
Figura 1 - Fluxograma mostrando as etapas do experimento.

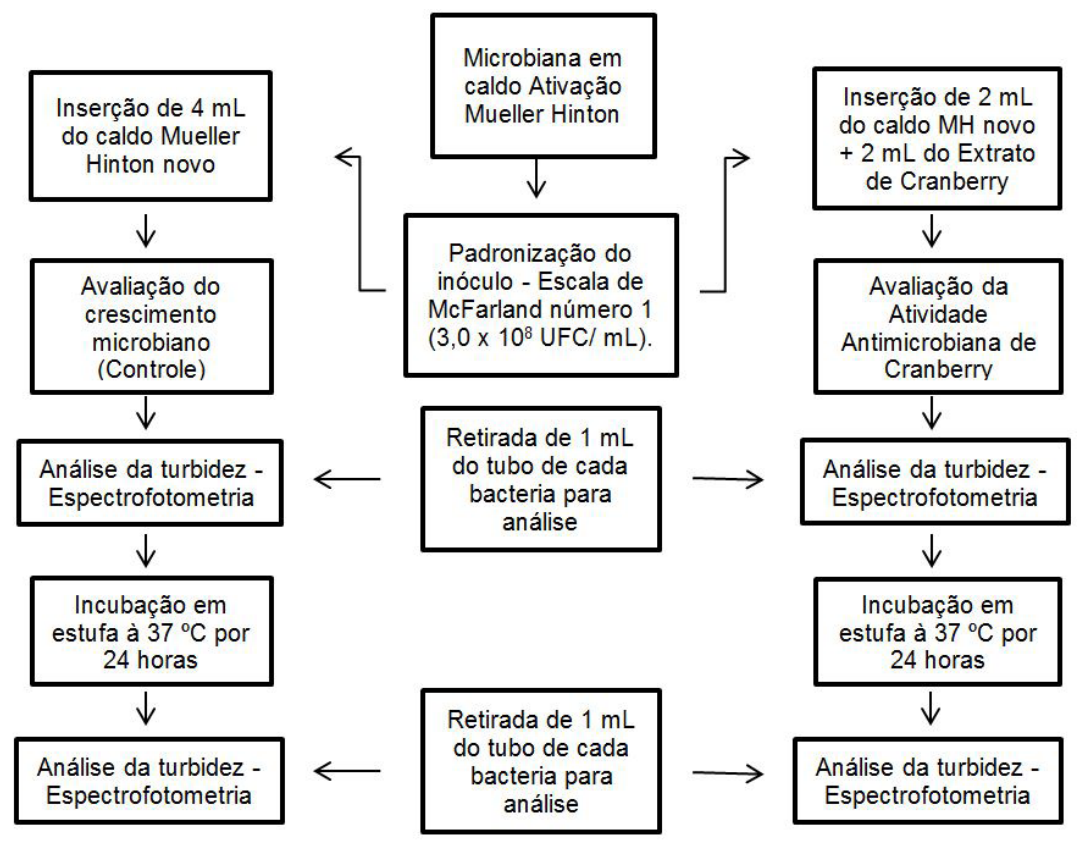

Fonte: Os autores.

A Densidade Ótica do caldo MH e MH com extrato de cranberry também foram aferidas e utilizadas como fator de correção, em função da cor.

\subsection{Testes estatísticos}

Para análise estatística, o software utilizado foi o BioEstat 5.0, no qual foram realizadas as médias DO de 3 repetições e Teste $t$ de Student. 0 teste $t$ de Student possibilita verificar a presença de significância estatística entre as médias dos dois tratamentos (Controle e Extrato). Como parâmetro de significância, o nível estabelecido foi $p<0,05$.

\section{RESULTADOS E DISCUSSÃO}

O Teste $t$ de Student indicou que o extrato de cranberry exerceu efeito de inibição nas bactérias Staphylococcus aureus, Klebsiella pneumoniae, Escherichia coli, Serratia marscecens e Enterococcus faecalis, indicado pela significância estatística $(p<0,05)$. Os valores de $p<0,01$ representam uma diferença mais significativa. No entanto, o extrato de cranberry não inibiu as bactérias Pseudomonas aeruginosa, Proteus mirabilis e Enterococcus faecium, indicado pelos valores de $\mathrm{p}$ maiores que o nível de significância proposto $(p>0,05)$, conforme Tabela 1. 
Tabela 1 - Significância estatística no crescimento microbiano entre o tratamento controle e o extrato de cranberry, em nível de $\mathrm{p}<0,05$.

\begin{tabular}{|c|c|c|c|c|}
\hline Bactérias & Tratamento & Média de 3 Repetições & $\mathbf{T}$ & $\mathbf{p}$ (unilateral) \\
\hline \multirow{2}{*}{ S. aureus } & Controle & 1.134 & 33.222 & $<0.01$ \\
\hline & Extrato & 0.030 & & \\
\hline \multirow{2}{*}{ K. pneumoniae } & Controle & 1.4083 & 3.9501 & 0,0292 \\
\hline & Extrato & 0.7633 & & \\
\hline \multirow{2}{*}{ E. coli } & Controle & 1.0463 & 15.255 & $<0.01$ \\
\hline & Extrato & 0.2607 & & \\
\hline \multirow{2}{*}{ S. marscecens } & Controle & 1.629 & 7.3548 & $<0.01$ \\
\hline & Extrato & 0.2553 & & \\
\hline \multirow{2}{*}{ E. fecalis } & Controle & 1.025 & 15.5691 & $<0.01$ \\
\hline & Extrato & 0.1627 & & \\
\hline \multirow{2}{*}{$P$. aeruginosa } & Controle & 1.2487 & -0.602 & 0,0784 \\
\hline & Extrato & 1.346 & & \\
\hline \multirow{2}{*}{ P.mirabilis } & Controle & 0.9473 & -1.7394 & 0,0784 \\
\hline & Extrato & 1.247 & & \\
\hline \multirow{2}{*}{ E. faecium } & Controle & 0.5217 & 1.8015 & 0.1067 \\
\hline & Extrato & 0.0317 & & \\
\hline
\end{tabular}

Fonte: Os autores.

Os resultados deste trabalho são semelhantes aos relatados por Cesoniene; Jasutiene; Sarkinas (2009), que obtiveram resultados significativos na inibição de uma ampla variedade de bactérias patogênicas, incluindo Escherichia coli, Staphylococcus aureus e Enterococcus faecalis, confirmando propriedades antimicrobianas do extrato de cranberry. Contudo, Gupta et al. (2007) mencionam a importante dependência da dose de proantocianidinas, evidenciando a relação da dose com os efeitos antimicrobianos causados.

A inibição da Escherichia coli também foi encontrada por Ahuja; Kaack; Roberts (1998), Liu et al. (2006) e Tao et al. (2011). Ahuja; Kaack; Roberts (1998) e Liu et al. (2006) sugerem que essa inibição acontece devido ao cranberry alterar a morfologia bacteriana através da inibição da expressão de fimbrias por Escherichia coli. Segundo os autores, a alteração seria a responsável pela redução da adesão fimbrial, um dos mecanismos utilizados para o estabelecimento de micro-organismos no trato urinário. Entretanto, os estudos genéticos e morfológicos se tornam necessários para confirmar as sugestões.

Tao et al. (2011), por meio de estudos in vitro, obtiveram efeito sobre a adesão de $E$. coli em culturas com amostras de urina de voluntários que consumiram o suco de cranberry. Estas amostras foram adquiridas de duas em duas horas, fazendo com que a concentração dos metabólitos do suco encontrados na urina fosse aumentando com o tempo. Os autores obtiveram resultados crescentes na inibição da adesão de acordo com o tempo e concentração dos metabólitos de cranberry, correspondendo com a discussão de Gupta et al. (2007). Dessa forma, os resultados são indício de que o potencial de inibição pode ser intensificado com o uso contínuo e constante do cranberry.

Contudo, esses resultados contrariam os obtidos por Lee et al. (2010), que apesar de observarem a atividade antimicrobiana do cranberry sobre Escherichia coli, o mesmo efeito não foi mais observado após 2-6 horas. Entretanto, a atividade antimicrobiana foi encontrada sobre a Klebsiella pneumoniae, mesmo passado esse período, confirmando os resultados inibitórios de nosso estudo. 
Hidalgo et al. (2011) fundamentaram o potencial preventivo do cranberry encontrado em seus resultados por meio da associação das proantocianidinas (PACs) com a limitação de ferro, afirmando que as PACs atuam como quelante de ferro. Com isso, indagou sobre a capacidade de quelação ser importante, pois também pode dificultar a formação de biofilme e influenciar nas propriedades anti-adesivas. Contudo, apesar dos resultados dos autores demonstrarem efeitos sobre Escherichia coli, seu crescimento não foi inibido pela atuação das proantocianidinas. Entretanto, pesquisas sobre o mecanismo de ação das proantocianidinas se tornam necessários para confirmar se a quelação é um fator considerável sobre a formação de biofilme e propriedades adesivas.

Os estudos da ação do cranberry realizado por Harjai; Gupta; Sehgal (2014) in vitro demonstraram que o cranberry é benéfico na prevenção de infecções do trato urinário (ITU). Entre os efeitos benéficos citados pelos autores, estão: redução na formação de biofilme na superfície do cateter, produção de sinais "quorum sensing" e fatores de virulência. Na pesquisa foi observada a diminuição da hidrofobicidade de superfície celular e adesão às células epiteliais, dois mecanismos de adesão que podem ser utilizados por bactérias. Contudo, os efeitos mencionados pelos autores foram encontrados em Pseudomonas aeruginosa, contrariando os resultados obtidos em nosso estudo, em que o efeito inibitório observado em $P$. aeruginosa não foi significativo. Entretanto, cepas diferentes apresentam comportamentos distintos, indicando que a cepa utilizada pode ter influenciado nos efeitos demonstrados pelos autores.

Um efeito de inibição significativo sobre Proteus mirabilis também não foi demonstrado em nossos resultados, corroborando o trabalho de McCall et al. (2013), que demonstraram que Proteus mirabilis não teve seu crescimento inibido pelo extrato de cranberry. Entretanto, seus estudos mostram que, apesar de não afetar o crescimento, o extrato dificulta a motilidade de $P$. mirabilis e reduz a expressão de importantes fatores de virulência.

\section{CONCLUSÃo}

O Extrato de Cranberry apresentou atividade antimicrobiana sobre as bactérias Staphylococcus aureus, Klebsiella pneumoniae, Escherichia coli, Serratia marscecens e Enterococcus faecalis, confirmando seu efeito benéfico em infecções urinárias.

No entanto, não teve efeito inibitório significativo sobre Pseudomonas aeruginosa, Proteus mirabilis e Enterococcus faecium. 
Avaliação da presença de tags intratubular na região apical de dentes anteriores unirradiculares, variando a técnica de manipulação do cimento resilon

\section{REFERÊNCIAS}

AHUJA, S.; KAACK, B.; ROBERTS, J. Loss of fimbrial adhesion with the addition of Vaccinum macrocarpon to the growth medium of P-fimbriated Escherichia coli. The Journal of Urology, v. 159, n. 2, p. 559-562, 1998.

ARMBRUSTER, C. E.; MOBLEY, H. L. T. Merging mythology and morphology: the multifaceted lifestyle of Proteus mirabilis. Nature Reviews Microbiology, v. 10, n. 11, p. 743-754, 2012.

ARMBRUSTER, C. E. et al. Arginine promotes Proteus mirabilis motility and fitness by contributing to conservation of the proton gradient and proton motive force. MicrobiologyOpen, v. 3, n. 5, p. 630-641, 2014.

ČESONIENĖ, L.; JASUTIENĖ, I.; ŠARKINAS, A. Phenolics and anthocyanins in berries of European cranberry and their antimicrobial activity. Medicina (Kaunas, Lithuania), v. 45, n. 12, p. 992 - 999, 2009.

CHEN, H.; ZUO, Y.; DENG, Y. Separation and determination of flavonoids and other phenolic compounds in cranberry juice by high-performance liquid chromatography. Journal of Chromatography A., n. 913, p. 387-395, 2001.

DEBNATH, I. et al. The cpx stress response system potentiates the fitness and virulence of uropathogenic Escherichia coli. Infection and Immunity, v. 81, n. 5, p. 1450-1459, 2013.

DESSİ, A.; ATZEI, A.; FANOS, V. Cranberry in children: prevention of recurrent urinary tract infections and review of the literature. Revista Brasileira de Farmacognosia, v. 21, n. 5, p. 807-813, 2011.

DIARRA, M. S. et al. In vitro and in vivo antibacterial activities of cranberry press cake extracts alone or in combination with $\beta$-lactams against Staphylococcus aureus. BMC Complementary and Alternative Medicine, 2013.

EROL, A.; ÇOBAN, S.; TEKIN, A. A Giant Case of Pyonephrosis Resulting from Nephrolithiasis. Case Reports in Urology, 2014.

GARZA-RAMOS, U.; SILVA-SÁNCHEZ, J.; MARTÍNEZ-ROMERO, E. Genética y genómica enfocadas en el estudio de la resistencia bacteriana. Salud Pública de México, v. 51, p. 439-446, 2009.

GUAY, D. R. P. Cranberry and Urinary Tract Infections. Drugs, v. 69, n. 7, p. 775-807, 2009.

GUPTA, K. et al. Cranberry products inhibit adherence of $p$-fimbriated Escherichia coli to primary cultured bladder and vaginal epithelial cells. The Journal of Urology, v. 177, n. 6, p. 2357-2360, 2007.

HARJAI, K.; GUPTA, R. K.; SEHGAL, H. Attenuation of quorum sensing controlled virulence of Pseudomonas aeruginosa by cranberry. The Indian Journal of Medical Research, v. 139, n. 3, p. 446-453, 2014.

HEAD, K. A. Natural approaches to prevention and treatment of infections of the lower urinary tract. Alternative Medicine Review, v. 13, n. 3, p. 227-244, 2008.

HIDALGO, G. et al. Induction of a state of iron limitation in uropathogenic Escherichia coli CFT073 by cranberry-derived proanthocyanidins as revealed by microarray analysis. Applied and Environmental Microbiology, v. 77, n. 4, p. 1532-1535, 2011. 
HISANO, M. et al. Cranberries and lower urinary tract infection prevention. Clinics, v. 67, n. 6, p. 661 667, 2012.

HOWELL, A. B. et al. Dosage effect on uropathogenic Escherichia coli anti-adhesion activity in urine following consumption of cranberry powder standardized for proanthocyanidin content: a multicentric randomized double blind study. BMC Infectious Diseases, 2010.

JOHNSON B. J. et al. Impact of cranberry on Escherichia coli cellular surface characteristics. Biochemical Biophysical Research Communications, v. 377, n. 3, p. 992-994, 2008.

LAVIGNE, J. P. et al. In-vitro and in-vivo evidence of dose-dependent decrease of uropathogenic Escherichia coli virulence after consumption of commercial Vaccinium macrocarpon (cranberry) capsules. Clinical Microbiology and Infection, v. 14, n. 4, p. 350-355, 2008.

LEE, Y. L. et al. Anti-microbial activity of urine after ingestion of cranberry: a pilot study. Evidence-based Complementary and Alternative Medicine, v. 7, n. 2, p. 227-232, 2010.

LIU, Y. et al. Role of cranberry juice on molecular-scale surface characteristics and adhesion behavior of Escherichia coli. Biotechnology and Bioengineering, v. 93, n. 2, p. 297-305, 2006.

LIU Y. et al. Cranberry changes the physicochemical surface properties of E. coli and adhesion with uroepithelial cells. Colloids Surf B Biointerfaces, v. 65, n. 1, p. 35-42, 2008.

LIVERMORE, D. M. Bacterial resistance: origins, epidemiology, and impact. Clinical Infectious Diseases, 2003.

LOGUERCIO, A. P. et al. Atividade antibacteriana de extrato hidro-alcoólico de folhas de jambolão (Syzygium cumini (L.) Skells). Ciência Rural, v. 35, n. 2, p. 371-376, 2005.

MAGARIÑOS, H. L. E. et al. In vitro inhibitory effect of cranberry (Vaccinium macrocarpum Ait.) juice on pathogenic microorganisms. Applied Biochemistry and Microbiology, v. 44, n. 3, p. 300-304, 2008.

McCALL, J. et al. Cranberry impairs selected behaviors essential for virulence in Proteus mirabilis HI4320. Canadian Journal of Microbiology, v. 59, n. 6, p. 430-436, 2013.

NICOLLE, L. E. Catheter associated urinary tract infections. Antimicrobial Resistance and Infection Control, 2014.

O'MAY, C.; TUFENKJI, N. The swarming motility of Pseudomonas aeruginosa is blocked by cranberry proanthocyanidins and other tannin-containing materials. Applied and Environmental Microbiology, v. 77, n. 9, p. 3061-3067, 2011.

SMALL, E. North American Cornucopia: Top 100 Indigenous Food Plants. CRC Press, 2013, 793 p.

SOUZA, A. C. S. et al. Cateterismo urinário: conhecimento e adesão ao controle de infecção pelos profissionais de enfermagem. Revista Eletrônica de Enfermagem, v. 9, n. 3, p. 724-735, 2007.

SU X.; HOWELL, A. B.; D'SOUZA D. H. Antibacterial effects of plant-derived extracts on methicillin-resistant Staphylococcus aureus. Foodborne Pathogens and Disease, v. 9, n. 6, p. 573-578, 2012. 
Avaliação da presença de tags intratubular na região apical de dentes anteriores unirradiculares, variando a técnica de manipulação do cimento resilon

TAO, Y. et al. Oral consumption of cranberry juice cocktail inhibits molecular-scale adhesion of clinical uropathogenic Escherichia coli. Journal of Medicinal Food, vol. 14, p. 739-745, 2011.

WANG, C. H. et al. Cranberry-containing products for prevention of urinary tract infections in susceptible populations: a systematic review and meta-analysis of randomized controlled trials. Archives of Internal Medicine, v.172, n.13, p. 988-996, 2012. 